\title{
LA PARTICIPACIÓN DE LOS ENTES INOBSERVABLES EN EL PROBLEMA CUERPO-MENTE. ARMONÍA ENTRE EL INTELECTO Y LA NATURALEZA ${ }^{1}$
}

The participation of the unobserbvable entities in the mind-body problematic. Harmony between intelect and nature

\author{
Alex Espinoza Verdejo* \\ Marcelo Díaz Soto**
}

Resumen

En este artículo se analiza la participación de los entes inobservables o teóricos en las teorías filosóficas cuyo contenido es el problema de la relación entre el cuerpo y la mente. Las concepciones sustancialistas, fisicalistas, conductistas filosóficas y escépticas son las fuentes de inspiración y desde las cuales se busca determinar la pertinencia o falta de pertinencia de los entes teóricos en el problema cuerpo-mente.

Palabras clave: Entes inobservables, sustancialismo, fisicalismo, conductismo filosófico.

Abstract

The object of this essay is to analyze the role played by unobservable or theoretical entities in the philosophical theories whose content is the mind-body problem. Substantialism, physicalism, philosophical behavioursm, and scepticism are the main sources of inspiration, and it is from them that I try to determine the pertinence or lack of pertinence of theoretical entities for the mind-body problem.

Key words: Unobservable entities, substancialism, physicalism, philosophical behaviour.

La historia de lo que hoy denominamos conocimiento científico sugiere que no podemos obtener logros exitosos en el campo de la inteligibilidad si no se construyen, con la ayuda de la imaginación, cuerpos teóricos bien definidos que den cuenta de los procesos o de las ontologías inobservables. Por ejemplo, hablamos de los quarks en física, de las fuerzas organizadoras en biología. Los poderes racionales en su lucha constante contra la amenaza del escepticismo se tranquilizan en la medida en que las construcciones teóricas se ajusten a las demandas de la comprensión de los fenómenos, así podríamos hablar de una armonía del intelecto y la naturaleza. Más que buscar constataciones empíricas buscamos comprender; las comprensiones se determinan a través de los constructos teóricos. Esto nos hace pensar que en las ciencias, la mayoría de las veces, la inteligibilidad debe admitir la participación de los inobservables, que podríamos llamar los "inobservables inteligibles". La exigencia del conocimiento

\footnotetext{
${ }^{1}$ Este artículo forma parte del Proyecto de Investigación Mayor de la Universidad de Tarapacá de Arica, Código 3732-10 y del Proyecto DICYT (Dirección de Investigación en Ciencia y Tecnología de la Universidad de Santiago de Chile), Código: 031053DS.
} 


\section{Alex Espinoza y Marcelo Díaz}

científico a las explicaciones teóricas es que en algún momento pasen a formar parte del mundo de los observables. Las preguntas que emergen de estos inobservables inteligibles son: ¿Cómo es posible el ajuste entre la teoría y la realidad? ¿Buscamos sistemas isomórficos? Si es efectivo que buscamos sistemas isomórficos, entonces ¿Qué factores son determinantes en ellos? Los sistemas isomórficos necesitan de los modelos analógicos, como en la ciencia física lo es el péndulo y las fuerzas gravitatorias, la estructura del átomo y el sistema solar; en biología y ciencia de la información: el cerebro humano y el computador.

Si el mundo está plagado de formas semejantes y que son factores determinantes en el mundo de los objetos, entonces estas formas favorecen las analogías en las ciencias, las formas constituyen una buena estrategia para explicar los mecanismos naturales escondidos a las constataciones empíricas. Aquí detendremos nuestra reflexión. Ahora relacionemos este tema con el problema filosófico cuerpo-mente.

EL LEGADO DE LOS PENSADORES CLÁSICOS: ENTRE EL DUALISMO PLATÓNICO Y EL MONISMO ARISTOTÉLICO

El dualismo platónico propone la idea de que el hombre posee una facultad integradora de todas las experiencias. Además, propone que el mundo está compuesto por objetos llenos de cualidades. De esta manera, el hombre se convierte en un cazador de cualidades, las cualidades de los objetos tienen sentido si son percibidas por esta facultad integradora llamada espíritu. Según el pensamiento platónico, el mundo espiritual está constituido por entidades que sólo habitan en los espacios de nuestra intimidad humana, el hombre es el único ser que tiene acceso privilegiado a este mundo, al mundo del espíritu.

En este mundo, las entidades gozan de una perfección máxima, el mundo Ideal sirve de patrón, desde el cual el mundo real y concreto debe medir su grado de perfección. Estas Ideas se corresponden con el legado de herencias innatas. Así, también podríamos afirmar que el espíritu en el hombre es un legado heredado, por cuanto, el mundo de las Ideas requiere de un lugar donde pueda desplegarse dinámicamente. ${ }^{1}$ Uno de los problemas filosóficos que debe enfrentar el dualismo mente-cuerpo es aquel de la conexión causal. En otras palabras ¿Cómo es posible que algo que es definido como inmaterial o no-espacial y atemporal puede influir en algo que es material y temporal? Algunas explicaciones metafísicas, como la de Descartes, o la del mismo Platón, resuelven este problema acudiendo a la intervención divina en el sistema cognitivo.

El hilemorfismo aristotélico responde al problema de la conexión causal de los mundos platónicos. El Estagirita, contrario a la creencia platónica, se

\footnotetext{
${ }^{1}$ William Bechtel menciona algunas opiniones actuales que dan crédito a la idea del innatismo: Chomsky "argumentó que el conocimiento de las reglas sintácticas tenía que ser innato puesto que un niño no tiene experiencia suficiente para aprenderlas por inducción" (1991).
} 
centrará en la reflexión metafísica sobre las formas que dan identidad al mundo. Aristóteles considera que el hombre se encuentra rodeado de un mundo poblado de objetos y que la existencia de estos objetos depende, en última instancia, de su constitución material y con ello — afirma metafísicamente el Estagirita- que los objetos son identificables por su forma. La totalidad de los objetos con sus formas particulares dan sentido a la vida humana, los objetos tienen una forma que es propiamente natural a la materia. La forma yace en los mismos objetos, la forma es la esencia, la forma representa la estabilidad de las cosas. Las formas yacen en la misma realidad y no habitan en un mundo privilegiado del espíritu como afirmaba Platón.

En nuestros días, de acuerdo con la evolución del conocimiento, las formas corresponden a las regularidades testificadas por las ciencias; las cosas se rigen por cierto orden. Debemos destacar que hoy las leyes científicas son interpretadas bajo el positivismo y existe un acuerdo tácito para excluir toda interpretación mediada por la metafísica. Esta exclusión ha traído serios problemas al conocimiento científico representado en el problema filosófico de la inducción ¿Cómo es posible la universalización? ¿Es posible predicar la validez de las leyes para todos los mundos posibles? La captación de estas formas exige la existencia de una facultad organizadora. Aristóteles llamará a esta facultad intelecto, el intelecto será la "forma" del ser humano, es lo que le proporciona identidad; pero el ser humano también está dotado de un cuerpo. Así, Aristóteles concluye en el hilemorfismo, pues no podemos pensar el alma sin un cuerpo que la albergue, como tampoco podemos pensar en un cuerpo sin un alma.

En la historia de las ciencias cognitivas se han clasificado las teorías de Platón y de Aristóteles como representacionistas, vale decir, que la realidad puede ser conocida en toda su profundidad por el sistema cognoscitivo. En el fondo, se afirma que el espíritu o el espíritu-cuerpo es un receptáculo inerte, lo cual corresponde a la metáfora del espejo.

\section{EL CONDUCTISMO FILOSÓFICO, LÓGICO O ANALÍTICO DE GILBERT RYLE ${ }^{2}$}

Las teorías sustancialistas clásicas que fundamentan la existencia del espíritu en el hombre encontrarán un respaldo justificacionista en la época moderna; por ejemplo, pensemos en el racionalismo de Descartes. El dualismo cartesiano se

\footnotetext{
${ }^{2}$ El conductismo filosófico (Hempel, Ryle, Wisdom y en menor grado Wittgenstein) es una propuesta de cómo debe analizarse el lenguaje acerca de lo mental e incluye, especialmente en el caso de Ryle, la afirmación típicamente ontológica y materialista que los estados mentales internos, privados y accesibles directamente vía introspectiva son un mito, no existen. En cambio el conductismo en psicología es una propuesta metodológica sobre cómo hacer investigación psicológica ajustándose al método estándar que proviene de la ciencia natural. En sentido estricto, esta forma de conductismo no se pronuncia sobre el estatuto ontológico de los estados mentales internos, solamente dice de ellos que en caso de haberlos no son tratables científicamente por su carácter subjetivo. Cfr., M. Díaz (1989).
} 


\section{Alex Espinoza y Marcelo Díaz}

restablece en la intuición intelectiva. La existencia del cogito es una cuestión evidente y queda fuera de toda duda por muy metódica que ésta sea. Gilbert Ryle en El concepto de lo mental (1967) destruye el dualismo de Descartes; destrucción que ejecuta desde los dominios de la filosofía analítica. Ryle argumenta que cometemos un error categorial cuando atribuimos al hombre una facultad particular como es la del espíritu. Ryle realiza un análisis de la lógica del lenguaje natural e invita a pensar en el ejemplo de la visita que realiza un extranjero a la Universidad de Oxford. Ve edificios, laboratorios, casinos, jardines, etc., pero luego se pregunta ¿Dónde está la universidad? De este modo, el extranjero piensa que la universidad se representa en una ontología específica, por cuanto el concepto de universidad busca una hipostación. De igual modo a lo descrito sobre el concepto de universidad tratamos cartesianamente el concepto de espíritu. Al hombre -al que consideramos dotado de una inteligencia porque ejecuta actos inteligentes - le asignamos, además de un cuerpo, una entidad no-física, una entidad que generalmente llamamos espíritu, alma, mente, conciencia. Oponiéndose a esta doctrina cartesiana, Ryle afirma que nosotros somos sujetos dotados de propensiones, de disposiciones a comportarnos de diferentes maneras. De esta manera, sostiene la tesis que la inteligencia es una propiedad disposicional, es decir, somos seres inteligentes en la medida en que ejecutamos conductas inteligentes. Mirado lo anterior desde la lógica del lenguaje, de lo mental o espiritual, esto quiere decir que los conceptos mentales, los que empleamos para hablar de nuestras experiencias, sentimientos, pensamientos, etc., son conceptos disposicionales.

A partir de estos antecedentes podemos avalar la idea que Ryle simpatiza con las teorías conductistas de la mente. Para aclarar el concepto de disposición, concepto central por cierto en la propuesta de Ryle, podríamos pensar en la fragilidad o carácter quebradizo de un cristal ¿Si decimos de un cristal que es quebradizo estamos diciendo que está quebrado? No. Lo que estamos diciendo es que si se dan ciertas situaciones empíricas — como la de ser golpeado- el cristal se quebrará; es decir, la lógica de los enunciados que incluyen conceptos disposicionales es de carácter semi-hipotético, funcionan como una autorización para inferir que si se dan ciertas condiciones ocurrirá algo. Llevada esta reflexión al ámbito de los conceptos mentales esto quiere decir que podemos dar igual trato lógico a conceptos como los de inteligencia y de fragilidad. Sin embargo, como tempranamente lo hiciera ver Bertrand Russell en su comentario (1976) de ¿Qué es mente? de Ryle, su enfoque de lo espiritual es, curiosamente, compatible con el dualismo cartesiano. La razón de esta situación tan extraña surge cuando nos preguntamos acerca de cuál es la diferencia entre ser inteligente y ser frágil o quebradizo. La respuesta de sentido común es que, en el primer caso, estamos predicando algo de las mentes o espíritus y, en el segundo, lo estamos haciendo de los cuerpos. 
La pregunta que podríamos hacernos es si el conductismo analítico destruye la idea que sostienen los pensadores idealistas en cuanto a que la mente humana tiene una ontología especial. El conductismo es una vertiente del mecanicismo y las concepciones mecanicistas se originaron para terminar con las concepciones sustancialistas, espiritualistas y vitalistas en las ciencias. Una radicalización del punto de vista de Ryle fue el reduccionismo fisicalista o nivelador. En este enfoque, los estados y procesos mentales se identifican con procesos y estados físico-químicos en el cerebro.

\section{TEORÍA DE LA IDENTIDAD PSICO-FÍSICA O MENTE-CEREBRO}

La teoría de la identidad psico-física corresponde a una especie de reduccionismo fisicalista. Vale decir, existe una gama importante de fenómenos naturales cotidianos que podemos identificar con lo que la ciencia sostiene, como, por ejemplo — citando a Paul Churchland - el sonido es idéntico a la oscilación de una cuerda electromagnética, el relámpago es idéntico a una gran descarga de electrones entre las nubes y la tierra. Respecto a los estados mentales Churchland afirma: "Lo que ahora consideramos que son "estados mentales", argumenta el teórico de la identidad, son idénticos a estados cerebrales exactamente de la misma manera" (1992:51). Actualmente, la neurociencia es la disciplina que mejor se ve representada con esta teoría. Se piensa que la taxonomía de cada acto mental establecida por el sentido común se correlaciona con una taxonomía neuronal establecida por la neurociencia. En la actualidad, la identificación psiconeural es un objetivo de la neurociencia, pero no se ha logrado completar, lo que sí se ha logrado es establecer la correlación (excepto en cuestiones tan complicadas como la base neuronal de la conciencia).

\section{PARTICIPACIÓN DE LOS ENTES INOBSERVABLES EN EL DUALISMO SUSTANCIALISTA}

Dado que ya hemos proporcionado estos antecedentes, podemos plantearnos la siguiente pregunta ¿Cómo participan los entes inobservables en el dualismo sustancialista? Es evidente que sí existe una participación de ellos. Por una parte, se afirma que el cuerpo como macro-fenómeno es una entidad física que se determina mediante todas las estrategias desarrolladas por las llamadas ciencias fácticas. Por ejemplo, en psicología el cuerpo se ha estudiado con cierto éxito a través de los reduccionismos biológicos y conductuales, haciendo ver el triunfo de las estrategias positivistas. A este respecto, la participación de los entes inobservables es escasa, a no ser que estemos pensando en la biofísica, por cuanto, el desarrollo de estas ciencias es altamente teórico, sobre todo, en lo que respecta a la física.

Por otra parte, y contrariamente a la concepción fisicalista, la concepción espiritualista en el hombre nos acerca más a la participación de los inobservables en las formulaciones teóricas. El nous socrático o "el yo cartesiano", en cuanto teorías filosóficas, ponen de manifiesto la capacidad intuitiva del hombre. La intuición intelectual revela la existencia de una sustancia pensante. La existencia 


\section{Alex Espinoza y Marcelo Díaz}

del espíritu no es deducible de ningún sistema, sea material o inmaterial; la intuición revela el ser de las cosas en su totalidad. Así, el espíritu como un ente inobservable se autodefiniría como un sentimiento, sería el cogito cartesiano atrapado por la intuición intelectual, en lenguaje de Bradley sería la "mismidad", algo que es plenamente privado o íntimo. De lo anterior podemos afirmar que el constructo teórico del espíritu no admite ninguna prueba experimental física de su existencia. A lo más, podemos afirmar que existe indirectamente algo que nos hace sentir; pero este algo no es similar a nada o a ninguna cosa existente en el mundo real, entendiendo como mundo real aquel que está determinado por las coordenadas de tiempo y espacio. El espíritu como ente inobservable no acepta isomorfismos, con esta afirmación se está fijando la tesis que los modelos representacionistas del espíritu constituyen un sin sentido. El espíritu está vestido con el ropaje de todas las actividades humanas, pero al sacar todo el ropaje nos quedamos con un ente que ni siquiera es inobservable, los entes inobservables admiten lo posible de la observabilidad en principio. Los hombres de ciencia construyen entes inobservables a partir de ciertas manifestaciones indicadoras de un existente posible. Pensemos en los átomos de los pensadores griegos como el de Leucipo y Demócrito o la existencia de la gravitación universal. Tenemos experiencia de la gravedad, pero no tenemos experiencia directa de su ontología. El espíritu se propone como un principio o como la premisa más importante y apreciada, un principio que podríamos llamar axiomático.

\section{PARTICIPACIÓN DE LOS ENTES INOBSERVABLES EN EL HILEMORFISMO ARISTOTÉLICO}

En el caso del monismo metafísico de Aristóteles podemos saborear la idea que el espíritu, en principio, admitiría la observabilidad y que está determinado por el mundo de la materia. La materia no existe sin una forma definida y es ésta la que le da identidad. El espíritu es la forma del cuerpo, el espíritu no lo podemos pensar como ajeno al cuerpo. En la filosofía de Aristóteles, la forma es un observable. De esta manera, podríamos afirmar que la forma, en tanto que espíritu, se muestra por las acciones, la metafísica caja negra de Skinner cobraría sentido en el esquema de la metafísica aristotélica.

El monismo metafísico de Aristóteles da cuenta de la continuidad del ser. La opción que realiza por la continuidad del ser lo pone fuera del alcance de varios problemas y críticas que normalmente se han dirigido en contra de las tendencias dualistas. Con esta elección se evita uno de los problemas más importantes: el interaccionismo cuerpo-espíritu ¿Cómo podemos justificar que el espíritu, siendo algo inmaterial y atemporal, pueda influir sobre algo que es material y temporal? o en lenguaje platónico ¿Cómo es posible que el espíritu siendo toda perfección pueda gobernar un mundo de imperfecciones? La teoría hilemórfica aristotélica escapa abiertamente de estos problemas. Pensar el espíritu, en tanto que forma del cuerpo o de la materia, indica claramente la continuidad en el hombre. La teoría emergentista en las ciencias naturales ve 
la continuidad del ser: de los seres inorgánicos emergen los seres orgánicos y de éstos los animales y, por último, el hombre y en él la espiritualidad. Jules Lachelier (1961) ve esto como una inducción natural, la naturaleza debe aceptar un telos organizador: creó el espíritu para que de esta manera pueda pensarse a sí misma, la naturaleza es inteligente. Estas ideas telefinalistas de Lachelier son cuestionadas por François Jacob (1970) para quien la naturaleza es un resultado de un pegoteo de evoluciones, la naturaleza es más bien un collage. Frente a estas polémicas epistemológicas, es obvio que la posición filosófica emergentista de Lachelier es más bien aristotélica. Así, podríamos afirmar que la forma es una cualidad emergente de la materia, de modo tal que el espíritu es una cualidad emergente de la materia.

Una manera de tener más elementos para pensar y aclarar el hilemorfismo aristotélico consiste en recurrir a las ideas que René Thom ha expuesto en Esquisse d'une sémiophysique (1988): que los conceptos de saillances y prégnances son los ancestros gestálticos de los conceptos de forma y fuerza respectivamente. La forma saillante es cualquier forma individual que ocupa una región definida del espacio-tiempo. Las prégnances son las formas o entidades no localizadas, emitidas y recibidas por las saillances. ${ }^{8} \mathrm{Si}$ acercamos las ideas de saillance y la prégnance al problema cuerpo-mente, ¿Ayudan a tener una mejor comprensión del problema cuerpo-mente en el hombre? o ¿Ayudan a mejorar la comprensión del hilemorfismo aristotélico? Al afirmar que la saillance es una forma definida y la prégnance es una cierta emisión de cualidades que la forma recepciona, creo que podríamos - tal vez - aproximar estos conceptos con la consideración que la mente o espíritu son disposiciones, en otras palabras, podríamos esperar algo provocador: que estos conceptos nos acercan a la concepción conductista y analítica de Gilbert Ryle. La pregnance sería equivalente a la disposición que tiene el espíritu para referirse a las cosas, la saillance es el lugar de donde emanan las disposiciones.

PARTICIPACIÓN DE LOS ENTES INOBSERVABLES EN EL CONDUCTISMO FILOSÓFICO

Como ya hemos afirmado, el conductismo se deriva del mecanicismo que está guiado por la idea de que no existen entidades extrañas al sistema que

\footnotetext{
8 "La mayor parte de las saillances descubiertas por los animales o por los hombres desfilan delante de los organismos sin dejar trazas durables. Pero existen formas visibles, auditivas, olfativas, etc., que - gracias a su constitución y a la disposición del organismo - están dotadas de una significación privilegiada por el organismo desde el cual el comportamiento está afectado: la presa para el depredador, el compañero sexual durante el periodo apropiado, etc. La prégnance es el carácter específico de estas formas significativas. Las saillances y las prégnances son compatibles porque las primeras pueden estar investidas por una significación de un valor reservándose una prégnance, y la nueva forma puede, en su lugar, transmitir la prégnance a otras saillances, Podríamos pensar en la transmisión de una infección por contagio. Cfr., Miguel Espinoza (1998-2002).
} 


\section{Alex Espinoza y Marcelo Díaz}

abriga todo su dinamismo. Así, se podría afirmar, desde una perspectiva conductista, que la mente humana existe porque ella expresa una serie de propensiones, tendencias que - en su gran mayoría - están estandarizadas y, así, los psicólogos pueden afirmar si se está en un nivel de normalidad o no, el criterio de normalidad hace referencia a una unidad determinada, la normalidad es un promedio de un todo. Pero ¿Qué significa un todo?, ¿Qué significa una unidad? El conductismo se ve en la obligación de aceptar una unidad organizadora, aunque la unidad organizadora sea el todo. Las funciones de cada pieza del reloj hacen referencia, en última instancia, a un reloj y las funciones de cada pieza que componen al humano también forman un todo; a esto los vitalistas llamarán espíritu o energía organizadora. El conductista si no recurre a esta unidad, entonces, se queda con una caja negra; con un inobservable al igual que el dualista sustancialista; con una entidad vacía desde el punto de vista ontológico. El conductista que no admite un inobservable se aleja del principal objetivo de nuestra racionalidad que consiste en entender los fenómenos. Los psicólogos que tienen el poder curativo y que esquivan los inobservables, permanecen en la ininteligibilidad, pues no tienen la capacidad de entender qué pasó realmente en el momento de la cura, como también sucede lo mismo con el físico que estudia la emanación de energía.

\section{LA PARTICIPACIÓN DE LOS ENTES INOBSERVABLES EN LA TEORÍA DE LA IDENTIDAD: MENTE-CEREBRO}

En el caso de Jean Pierre Changeaux, la teoría de la identidad cumple un rol eliminativista de la participación de los entes inobservables en las ciencias cognitivas, pero además ella es respetuosa de dicha participación por cuanto los admite como constituyentes necesarios en nuestra cultura. Si los objetos mentales son reemplazados por actividades electro-químicas de poblaciones neuronales, manifiesta claramente la idea de eliminar la participación de los entes inobservables, pero vemos que ésta es en un solo sentido, vale decir, elimina sólo aquellos entes que tienen una connotación espiritualista, siendo que por el hecho de ser "identidad" la relación eliminativa debería ser en ambos sentidos. La teoría psicofísica de la identidad es leída en un solo sentido. Si esto es así, entonces, pareciera ser una especie de reduccionismo materialista, pero en el caso contrario, vale decir, reducir los fenómenos biológicos a fenómenos espiritualistas, es una tesis que los teóricos con tendencias materialistas no estarían dispuestos a compartir. Porque si bien los materialistas reconocen la realidad de los estados mentales internos y el papel causal que éstos tienen sobre la conducta, identifican tales estados con los estados físico-químicos del cerebro, es decir, con algo material, de manera que la conducta es resultado de lo que sucede internamente en el cerebro; en cambio los espiritualistas o idealistas, especialmente en su versión pampsiquista, asumen que lo real está completamente constituido por átomos espirituales. Se 
podría decir que por definición la ontología del materialismo ("todo es material) es incompatible con la ontología del espiritualismo ("todo es espiritual o mental").

Podríamos, además, agregar que la teoría de identidad parte del supuesto que existe una realidad fenoménica material y otra realidad no material, pero hoy sabemos que el concepto de materia está ampliamente puesto en cuestionamiento, se prefiere hablar de unidad energética y no atómica, el concepto de energía es diferente al de materia, ambos conceptos no comparten las mismas categorías de análisis.

\section{HACIA UNA CONCEPCIÓN ESCÉPTICA MODERADA DE HUME DEL PROBLEMA CUERPO-MENTE Y LA SOLUCIÓN DE THOMAS REID}

David Hume es considerado entre los filósofos más influyentes en la elaboración del empirismo moderno. Su postura empirista extrema lo llevan finalmente a aceptar un escepticismo especial, que él mismo denomina mitigado. Por estas razones también ha sido un gran inspirador del movimiento positivista lógico y también se considera el autor del descubrimiento del llamado problema de la inducción. Para Hume, el conocimiento en el hombre se forma gracias a la relación que tiene el sistema cognitivo con la realidad. Hume fija el punto de conexión en la impresión (impressions), para luego pasar a las percepciones y, luego, a las llamadas ideas simples, para que así, finalmente, se pase a las ideas complejas. Hume determina estos procesos a través de la asociación, creando en el hombre los hábitos y las costumbres (custom), vale decir, los sistemas de creencias. Es así como Hume concluye en el escepticismo moderado: no existe la certeza del conocimiento, pero los sistemas de creencias ayudan a suavizar el escepticismo y con ello el problema de la inducción encontraría una solución parcial de tipo psicológica y sociológica, pero no formal. Para Hume, la imaginación es la facultad que operacionaliza esta dinámica para concluir en los sistemas de creencias; a su juicio, el conocimiento no es más que un haz de experiencias, no hay un "yo" organizador, sino que las experiencias se mediatizan por las creencias (belief). En algún sentido filosófico, la teoría del conocimiento o la gnoseología de Hume es enactivista, como aquella sostenida por Francisco Varela (1990): el sistema cognitivo y la realidad se co-determinan. De este modo, para Hume, la sobrevivencia es, en última instancia, la fuerza que lleva al hombre a adaptarse, cada vez, a las situaciones nuevas. En este sentido, Hume es un naturalista. Al dejar sin participación a los entes inobservables en la teoría del conocimiento, Hume estaría más próximo del funcionalismo, de allí que aparezca como fundador, también, de la fenomenología, porque se admite la idea de que la conciencia lo es en la medida en que está referida o intencionada; la intencionalidad viste de ropaje a la fuerza de la sobrevivencia del sistema biológico.

Thomas Reid (2004), leyendo a Hume - por quien tiene una simpatía especial- sugiere que para el sentido común son necesarios ciertos principios que tendrían el carácter de ser axiomáticos. Así, cree que eliminar del discurso del 


\section{Alex Espinoza y Marcelo Díaz}

sentido común el concepto del "yo" y reemplazarlo por el de haz de experiencia es una aberración. Estas ideas de Hume se fundan, sin lugar a dudas, en una especie de relativismo ontológico y epistemológico, las cosas viven en el tiempo y en el espacio, el ser cambia constantemente y todas las teorías que formulemos para dar cuenta de él son momentáneas. El sentido común exige el principio de identidad, en tanto ordenador del mundo.

Reid clasifica la filosofía de la mente de Hume como representacionista y concluye que éste acepta la teoría de las ideas simples, pero jamás la justificó. Reid problematiza la explicación de la intencionalidad que efectúa la teoría de las ideas en los términos siguientes: Supongamos que la teoría de las ideas simples fuese cierta, en tal caso, habría un sujeto, un objeto y una representación (del objeto). Esta representación debe tener algún tipo de relación con el objeto, ya sea de similitud o de causalidad. Por lo que el objeto es un signo que permite significarlo, para Aristóteles el signo que envuelve el significado es intuido, de allí emerge la representación. Pero sabemos que Hume esquiva el concepto de intuición y lo reemplaza por el de la captación de la unidad en lo percibido. Frente a esto, Reid cuestiona y afirma que la sola existencia del signo no es suficiente para dar cuenta de la intencionalidad del pensamiento. Para que un signo ejerza su función ha de ser interpretado. El sujeto es el intérprete de este signo, pero sólo puede interpretarlo en la medida en que posea concepciones previas del objeto representado. Entonces, el signo no consigue explicar la intencionalidad, porque lo presupone antes de la relación cognoscitiva sujeto-objeto-representación.

Estas ideas se explican, una vez más, cuando recurre al análisis de los principios del sentido común. Reid afirma que el sentido común no comprende la propuesta del "yo" cartesiano donde participan las ideas de las cosas; de este modo el concepto de abstracción es primordial, las ideas serían receptáculos vacíos en donde los objetos del mundo buscan encajar de la forma más perfecta posible, y que el acceso a este "yo" es por vía de la llamada intuición intelectual. Reid prefiere aceptar lo que el sentido común considera como cierto, el hombre accede al mundo a través de los juicios de las cosas y por aprehensiones vacías: "Todas las operaciones de los sentidos implican juicio o creencia, además de aprehensión simple (...) se sigue necesariamente que la aprehensión simple se obtiene resolviendo y analizando un juicio natural y original" (2004:88). En consideración a esto último, Reid estaría dispuesto a aceptar el tercer mundo de Popper (1979), el mundo de los contenidos lógicamente objetivos, un mundo cultural que contiene todas las manifestaciones de la creatividad humana. El mundo 1 sería el material o físico; el mundo 2 el psicológico o espiritual y el mundo 3 sería el resultado de los dos anteriores en un proceso de complejización creciente o de autoorganización de la materia inicial e inerte del mundo 1. En el centro de ese mundo 2 se 
encuentra el yo, que produce manifestaciones culturales, pero en sí mismo queda oculto, evasivo, como tan bien hicieran ver Hume y Ryle.

A modo de conclusión, podemos afirmar que los entes inobservables o teóricos en ciencia son necesarios para lograr el estado epistémico de la inteligibilidad, y como principio debemos aceptar la idea de que en algún momento los inobservables lleguen a ser observables, siendo esto último tarea de las ciencias. Así, existe una complementariedad entre el intelecto y la naturaleza.

También, hemos testimoniado que el dualismo sustancialista destruye la idea de la existencia de los entes inobservables; en otras palabras, no admite que los inobservables puedan convertirse en observables determinados científicamente. A lo anterior podemos agregar que el dualismo sustancialista no explica el interaccionismo causal cuerpo-mente. Por otra parte, el hilemorfismo aristotélico o monismo metafísico, acepta la participación de los inobservables en las teorías explicativas del mundo, solo que los inobservables se suponen como constitutivos propios de la misma naturaleza y que no podemos acceder a ellos, pero que en algún momento de la historia humana estos inobservables podrán revelarse en la realidad. Así, Aristóteles estaría de acuerdo en que la forma en el hombre, que es el espíritu, podrá mostrarse en las teorías del conocimiento por desarrollar.

Como propuesta alternativa al sustancialismo, las teorías conductistas y analíticas de la mente - especialmente la de Ryle - que no admiten la participación de los inobservables, deberá quedarse con la idea de que la mente no es lo que nos enseñó Descartes, una res cogitans, sino, más bien, un conjunto de propiedades disposicionales que se manifiestan en la conducta. Como vimos $-\mathrm{y}$ a contrapelo del conductismo que filosóficamente justificaba Ryle - si los estados mentales son disposiciones, nada impide resucitar el credo dualista cartesiano y concluir que las disposiciones pueden ser mentales o físicas. Con ello no solamente resurge el dualismo psico-físico, también, se reactiva el problema de la relación mente-cuerpo.

Otra manera de revisar el problema cuerpo-mente es analizando la teoría de la identidad mente-cerebro, teoría que celebra la idea que los fenómenos mentales se muestran en los fenómenos físicos o materiales, pero no admite la idea que los fenómenos cerebrales puedan representarse en fenómenos mentales. Los entes inobservables como constructos teóricos explicativos no tienen ninguna posibilidad y, en este sentido, el hombre es sólo un ser neuronal. La teoría de la identidad sirve sólo para eliminar toda concepción espiritualista de la mente, pero - en la relación de identidad - si eliminamos el sonido, eliminamos el trueno. Frente a las propuestas analizadas desde la perspectiva fisicalista o psicologista, no hemos querido dejar afuera las propuestas de carácter sociológica, el "yo" de Hume puede representarse, según Thomas Reid, 


\section{Alex Espinoza y Marcelo Díaz}

en la concepción o constructo que elabora el sentido común. En sentido Reid se aleja de una concepción escéptica del mundo, intento no logrado por Hume.

Finalmente, la pregunta que podemos dejar es ¿Qué pasaría si no incorporamos definitivamente la participación de los inobservables en ciencia? Muchas veces, la negativa es, más bien, de carácter ideológico e inconsciente. Debemos tener presente que bajo el problema cuerpo-mente la negativa se paga con la ininteligibilidad.

$$
\begin{array}{r}
\text { Universidad de Tarapacá* } \\
\text { Departamento de Psicologia y Filosofia } \\
\text { Avda.18 de Septiembre 222, Arica (Chile) } \\
\text { aespinoza@uta.cl } \\
\text { Universidad de Santiago de Chile** } \\
\text { Facultad de Humanidades } \\
\text { Departamento de Filosofia } \\
\text { Alameda Bernardo O'Higgins 3363. Santiago (Chile) } \\
\text { mdiazsoto@vtr.net }
\end{array}
$$

BIBLIOGRAFÍA

Bechtel, William. Filosofía de la mente y de las ciencias cognitivas. Madrid: Tecnos, 1991.

Changeux, Jean Pierre. L' homme neuronal. Paris: Fayard, 1983.

Churchrland, Paul M. Materia y conciencia. Barcelona: Gedisa, 1992.

Díaz, Marcelo. "Análisis crítico de aspectos de la teoría del lenguaje psicológico en Ryle y Wittgenstein", en Cuadernos de Humanidades, Facultad de Humanidades, Universidad de Santiago de Chile, No 2. (1989):1-80.

Espinoza, Alex. "Estudio de ontología: Las opciones ideológicas y el escepticismo de David Hume", en Revista Limite, No 9. (2002):27-47.

Espinoza, Miguel. Théorie de l'intelligibilité. Paris: Ellipse, 1998.

Herbert Bradley, Francis. Apariencia y realidad. Santiago de Chile: Edit. Universidad de Chile, 1961.

Jacob, François. La logique du vivant. Paris: Gallimard, 1970.

Lachelier, Jules. Du fondement de l'induction. Paris: Felix Alcan, 1916.

Popper, Karl. Objective Knowledge. Oxford: Clarendon Press, 1979.

Reid, Thomas. Investigación sobre la mente humana según los principios del sentido común. Madrid: Trotta, 2004.

Russell, Bertrand. “¿Qué es mente?”, en La evolución de mi pensamiento filosófico. Madrid: Alianza, 1976.

Ryle, Gilbert. El concepto de lo mental. Buenos Aires: Paidós, 1967.

Thom René. Esquisse d'une sémiophysique. Paris: InterEditions, 1988.

Varela, Francisco. Conocer. Las ciencias cognitivas: tendencias y perspectivas. Cartografia de las ideas actuales. Barcelona: Gedisa, 1990. 